\title{
HNRNPU Overexpression has Prognostic Value in Human Ovarian Cancer and Promotes Ovarian Cancer Cell Proliferation
}

\section{Ji Luo}

Nanchang University Second Affiliated Hospital

\section{Lulu Le}

Nanchang University Second Affiliated Hospital

\section{Xiaoli Tang}

Medical College of Nanchang University

\section{Yuanping Fu}

Nanchang University Second Affiliated Hospital

\section{Yueying Feng}

Nanchang University Second Affiliated Hospital

Fen Fu ( $\nabla$ fu_fen@163.com )

Nanchang University Second Affiliated Hospital

\section{Research}

Keywords: heterogeneous nuclear ribonucleoprotein U (HNRNPU), ovarian cancer (OC), somatic copy number alterations (SCNAs), cell proliferation.

Posted Date: November 24th, 2020

DOl: https://doi.org/10.21203/rs.3.rs-112897/v1

License: (9) This work is licensed under a Creative Commons Attribution 4.0 International License. Read Full License 


\section{Abstract}

Background: Ovarian cancer $(\mathrm{OC})$ is one of the most common gynecological malignancies and has the highest mortality rate worldwide. Therefore, the identification of novel targets and the development of more effective therapeutic treatments are essential.

Methods: We identified genes with somatic copy number alterations (SCNAs) amplification (AMP) according to The Cancer Genome Atlas (TCGA) OC data. We performed a meta-analysis of five independent $\mathrm{OC}$ datasets from the Gene Expression Omnibus (GEO) database. We retrieved cell cyclerelated genes from the online database GLAD4U. The associations of heterogeneous nuclear ribonucleoprotein $\mathrm{U}$ (HNRNPU) expression levels with clinicopathological factors and survival times were analyzed. In addition, we knocked down HNRNPU expression in two representative OC cell lines (OVCAR3 and ES2 cells) and then observed the proliferation capacity of OC cells.

Results: Analysis of the SCNA AMP genes, the transcriptome data of the GEO database and cell cyclerelated genes indicated that the HNRNPU gene was markedly upregulated in OC. High expression of HNRNPU in OC was related to SCNAs, poor survival and the OC proliferative molecular subtype. Based on the results in OVCAR3 and ES2 cells in vitro, we found that knocking down HNRNPU inhibited the proliferation of these two cell lines. Additionally, cell cycle arrest in the G1/S phase was found in the siHNRNPU cell group in vitro.

Conclusions: These results suggest that HNRNPU may serve as a candidate prognostic marker and a potential therapeutic target for OC.

\section{Background}

Ovarian cancer (OC), one of the most common gynecological malignancies, has the third highest incidence rate and the highest mortality rate among all malignancies of the female reproductive system[27, 30]. Worldwide, 239,000 new cases of OC and 152,000 deaths per year have been reported[5]. Although China has a rather low incidence rate, due to its large population, there were an estimated 52,100 new cases and 22,500 associated deaths in 2015[7]. While early-stage (FIGO I) OC has a five-year survival rate as high as $93 \%$, unfortunately, it is often diagnosed at an advanced stage owing to the lack of early symptoms and effective screening strategies[28]. Due to the lack of an effective targeted treatment strategy, the five-year survival rate for patients receiving a standard treatment regimen (debulking surgery and platinum/taxane-based chemotherapy) is only $30 \%$, and $5 \%$ of patients will develop resistance in six months[24]. Thus, investigations of the underlying molecular mechanisms, identification of novel biomarkers and recurrence prediction are urgent goals to improve the early diagnosis and prognosis of OC.

A large number of studies have shown that cell cycle disorders are closely related to human cancers. Moreover, our previous study found that the cell cycle may be the key pathway in the pathogenesis of $\mathrm{OC}[22]$. The poor DNA repair that can result from disruption of the G1/S phase may also lead to the 
genetically unstable phenotype typical of $\mathrm{OC}[1,15,16]$. These findings indicate that the cell cycle pathway may be closely related to the occurrence and development of OC.

Currently, somatic mutations have been demonstrated to be major factors in cancer development and progression[33]. In light of this, The Cancer Genome Atlas (TCGA) has been used to conduct comprehensive genomic research on $O C[6,26]$. The results show that somatic mutation patterns in $O C$ are rather unique compared to those of other tumor types, with somatic copy number alterations (SCNAs) as the main types. Recent omics screening has shown that there is a large number of SCNAs in the OC genome, and such genomic structural variations can modify the expression of effector genes in the affected region by changing their DNA copy numbers[9]. Subsequent functional analysis has indicated that the abnormal expression of these important functional genes (such as CCNE1 and MYC) is not only highly correlated with patient prognosis but also may serve as a promising target for precision treatment[2, 17]. Although SCNAs are the most important mutation pattern of OC, the mechanisms involved are still unknown.

In the present study, we identified SCNAs amplification (AMP) genes based on a TCGA term research. In addition, we obtained the $\mathrm{OC}$ transcriptome data from the Gene Expression Omnibus (GEO) database and identified the differentially expressed genes (DEGs) by a meta-analysis. To further explore the correlation between the cell cycle and gene AMP, we obtained cell cycle-related genes from the online database GLAD4U. Among these cell cycle-related genes, heterogeneous nuclear ribonucleoprotein $\mathrm{U}$ (HNRNPU) was one of the overexpressed genes in $\mathrm{OC}$ and was highly likely to be involved in $\mathrm{OC}$ progression. This study mainly focused on exploring the effects of HNRNPU in OC.

\section{Methods}

\section{Microarray data collection, comparison and meta-analysis}

Because the use of small sample sizes and different microarray analyses may lead to errors during the screening process, the results may not be completely reliable. A meta-analysis is a statistical analysis method that combines multiple studies to analyze a larger population to obtain more accurate and comprehensive results. GEO (https://www.ncbi.nlm.nih.gov/geo/) is a national public database that includes high-throughput gene expression, array-based and sequence-based data. In this study, relevant gene expression data were obtained from the GEO database (GSE6008, GSE18520, GSE26712, GSE27651 and GSE29450). Then, a meta-analysis of these datasets was performed to identify DEGs. In addition, the subtype classification data of GSE9891 were collected and analyzed, and the relationship between subtype classification and HNRNPU expression was studied.

\section{TCGA data extraction and analysis}

mRNA expression profiling data from TCGA were extracted from the UCSC database (http://xena.ucsc.edu/). Then, the OC samples downloaded from TCGA were categorized into four 
subtypes, immunoreactive, proliferative, differentiated and mesenchymal, according to the TCGA research team's criteria for classifying OC subtypes.

\section{Cell lines and cell culture}

The human OC OVCAR3 and ES2 cell lines were purchased from the Cell Resource Center of Shanghai Institute of Life Sciences, Chinese Academy of Sciences (Shanghai, China). The cells were grown in RPMI-1640 medium and Dulbecco's modified Eagle's medium (DMEM) (Invitrogen, Carlsbad, CA, USA), respectively. The cells were cultured in medium containing $10 \%$ fetal bovine serum (FBS) (Gibco, Grand Island, NY, USA) and $100 \mathrm{U} / \mathrm{mL}$ penicillin and streptomycin (Gibco, Grand Island, NY, USA) at $37^{\circ} \mathrm{C}$ with $5 \% \mathrm{CO}_{2}$. Then, the cells were harvested in the logarithmic growth phase for subsequent experiments.

\section{Transfection and RNA interference}

Transfections with small interfering RNAs (siRNAs) were performed using TurboFect Transfection Reagent (Thermo Scientific, Waltham, MA, USA) according to the manufacturer's protocol. siRNAs were synthesized by Shanghai Jima pharmaceutical technology (Shanghai, China). The siRNA sequences for human HNRNPU were as follows: siHNRNPU_\#1, CUGUGAGACUGAAGAUUAUTTAUAAUCUUCAGUCUCACAGTT and siHNRNPU_\#2, GCUGAGUGCUUUGAUGAAATTUUUCAUCAAAGCACUCAGCTT. The sequence of the control siRNA was UUCUCCGAACGUGUCACGUTTACGUGACACGUUCGGAGAATT.

\section{RNA purification and quantitative RT-PCR}

We used $1 \mu \mathrm{g}$ of total RNA extracted with TRIzol Reagent (Invitrogen, Carlsbad, CA, USA) for reverse transcription (RT) using the PrimeScript RT reagent Kit with gDNA Eraser (Perfect Real Time) (Takala Biomedical Technology, Beijing, China) according to the manufacturer's protocol. Quantitative RT

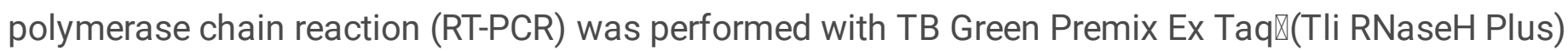
(Takala Biomedical Technology, Beijing, China) using an Applied Biosystems StepOnePlus Real-Time PCR System (Applied Biosystems, Foster City, CA USA). All reactions were conducted in triplicate. We calculated the relative mRNA expression by the comparative $\mathrm{Ct}$ method using glyceraldehyde-3phosphate dehydrogenase (GAPDH) as an internal control. The following primers were used for GAPDH: forward, 5'-TGACTTCAACAGCGACACCCA-3' and reverse, 5'-CACCCTGTTGCTGTAGCCAAA-3'. The following primers were used for HNRNPU: forward, 5'-GAGCATCCTATGGTGTGTCAAA-3' and reverse, 5'TGACCAGCCAATACGAACTTC-3'.

\section{Western blot analysis}

Briefly, cells were lysed with radioimmunoprecipitation assay cell lysis buffer (Solarbio Life Sciences, Beijing, China) containing $1 \mathrm{mM}$ phenylmethylsulfonyl fluoride (PMSF) and quantified using a bicinchoninic acid assay kit (Solarbio Life Sciences, Beijing, China). Equal amounts of protein $(20 \mu \mathrm{g})$ were separated by SDS-PAGE ( $8 \% \mathrm{gel})$ and then transferred to nitrocellulose membranes (Millipore, 
Billerica, MA, USA). The membranes were blocked for $1 \mathrm{~h}$ with $5 \%$ skimmed milk at room temperature and then incubated with primary antibodies overnight at $4^{\circ} \mathrm{C}$. The primary antibodies against HNRNPU $(1: 1000)$ and GAPDH $(1: 1,000)$ were purchased from ProteinTech Group (Chicago, IL, USA). After three washes in Tris-buffered saline with Tween-20, the membranes were incubated with secondary goat antirabbit or goat anti-mouse antibody (ProteinTech Group, Chicago, IL, USA) at a dilution of 1:1000 for $2 \mathrm{~h}$ at $37^{\circ} \mathrm{C}$. Finally, proteins were visualized with an enhanced chemiluminescent (ECL) detection solution (Millipore, Billerica, MA, USA).

\section{CCK-8 assay}

CCK-8 assays were performed using CCK-8 reagent (Solarbio Life Sciences, Beijing, China). A total of 3000 cells were seeded into a 96 -well culture plate and incubated for 1-6 days in an incubator at $37^{\circ} \mathrm{C}$ in $5 \% \mathrm{CO}_{2}$. One hundred microliters of prepared solution ( $10 \mu \mathrm{l} \mathrm{CCK-8} \mathrm{reagent} \mathrm{added} \mathrm{to} 90 \mu \mathrm{l}$ medium) was added to each well and incubated for $2 \mathrm{~h}$. The absorbance (OD) of each well at $450 \mathrm{~nm}$ was measured using a CMax Plus microplate reader (Molecular Devices, San Francisco, CA, USA). The growth curve was generated according to the OD values.

\section{Colony formation assay}

A total of 3000 cells were seeded into a 6-well culture plate and incubated for 2 weeks in an incubator at $37^{\circ} \mathrm{C}$ in $5 \% \mathrm{CO}_{2}$ until visible cell clones were observed. The clones were fixed with $4 \%$ formaldehyde (Sigma, St Louis, MO, USA) for 30 min and stained with $1 \%$ crystal violet (w/v) for 15 min before counting the number of colonies.

\section{5-Ethynyl-2'-deoxyuridine (EdU) staining assay}

Cells were further cultured for $48 \mathrm{~h}$ after transfection and labeled by EdU DNA Proliferation in vitro Detection (RiboBio, Guangzhou, China). We used phosphate-buffered saline (PBS) containing 4\% polyformaldehyde to fix the cells at room temperature for $30 \mathrm{~min}$. Then, we neutralized the cells with 2 $\mathrm{mg} / \mathrm{mL}$ deoxythymidylate, which was cleared with $0.1 \%$ Triton X-100 applied for $10 \mathrm{~min}$, followed by a PBS wash. Apollo 488 was applied for 30 min, and the nuclei were stained with Hoechst 33342 for 30 min. Subsequently, images were captured using a fluorescence microscope. DAPI-stained cells were regarded as total cells. The signal was activated by laser at a wavelength of $350 \mathrm{~nm}$, while the emitted light was collected in the blue channel (bandpass filter of 420-500 nm). We obtained the number of total cells by collecting and analyzing the target signatures at peak intensity, width and depth. The EdU-Apollo 488-stained cells were considered proliferating cells. The signal was activated by laser at a wavelength of $490 \mathrm{~nm}$, and emitted light was captured by the red channel to determine the number of proliferating cells.

\section{Analysis of cell cycle distribution using flow cytometry}

OVCAR3 and ES2 cells $\left(1 \times 10^{6}\right)$ treated with or without siHNRNPU were collected, fixed with $1500 \mu \mathrm{L}$ of $95 \%$ cold ethanol for $2 \mathrm{~h}$ or overnight according to the manual for the cell cycle detection kit (Keygen 
Biotech, Nanjing, China) and stored at $4^{\circ} \mathrm{C}$. After removal of the fixative with PBS, the cells were placed in a water bath, $2 \mu \mathrm{L}$ RNase A (50 $\mu \mathrm{g} / \mathrm{mL}$ final concentration) was added at $37^{\circ} \mathrm{C}$, and the cells were incubated for $30 \mathrm{~min}$. Furthermore, we incubated the cells with $25 \mu \mathrm{L}$ propidium iodide (PI) $(50 \mu \mathrm{g} / \mathrm{mL}$ final concentration) for 30 min in the dark. The cell cycle was analyzed with a BD Accuri C6 flow cytometer (Beckman Coulter, Fullerton, CA, USA) by red fluorescence set to a wavelength of $480 \mathrm{~nm}$.

\section{Statistical analysis}

Data were assessed using Student's t-test or one-way ANOVA followed by Fisher's multiple range test.

\section{Results}

\section{Selection of the target gene HNRNPU via bioinformatics analysis}

According to TCGA research network data[6], we identified 50 AMP regions (residual $q<0.05$ ) and 1,212 genes located in these regions. Subsequently, we collected OC transcriptome data for 422 samples (382 tumor tissues and 40 control tissues) from five GEO datasets (GSE6008, GSE18520, GSE26712, GSE27651 and GSE29450). Then, a meta-analysis of these five datasets was performed, and 6,735 DEGs were identified $(P$-value $<0.03)$. To further explore the correlation between the cell cycle and gene AMP, 1,243 cell cycle-related genes were obtained from the online database GLAD4U (http://bioinfo.vanderbilt.edu/glad4u/). We also identified 362 genes that have been reported in OC by GLAD4U. As shown in Fig. 1A, 24 unreported cell cycle-related genes were involved in AMP, of which 14 were upregulated genes (NCAPG2, HNRNPU, CSNK1D, DDA1, PAXIP1, CDCA4, CEP72, NPLOC4, GPS1, STK38, CAP1, CCND3, IGF1R, and POLH) and 10 were downregulated (NFYA, MAD2L1BP, NFYC, NUP153, $M C L 1, F B L, P I M 1, M Y C, C D C 5 L$, and HSP90AB1). Among these cell cycle-related genes, HNRNPU was one of the overexpressed genes (Fig. 1B).

\section{HNRNPU overexpression in OC is related to poor prognosis and SCNAs}

TCGA data showed that the expression level of HNRNPU was positively correlated with SCNAs, and AMP significantly increased the HNRNPUmRNA level (Fig. 2A). As shown in Fig. 2B, the expression level of HNRNPUin tumor tissues was higher than that in the control group, and the difference was statistically significant $(\mathrm{SMD}=1.27 ; 95 \% \mathrm{Cl}=0.38-2.16 ; P$-value $<0.01)$. Furthermore, we used Kaplan-Meier plotter (http://kmplot.com/analysis/) to analyze the prognostic value of the mRNA expression of HNRNPUin OC, and the results showed that the high expression of HNRNPU was related to a reduced survival time of patients with OC (Fig. 2C).

To further explore the relationship between the high expression of HNRNPU and the molecular subtype classification of OC, 298 samples downloaded from TCGA were divided into four molecular subtypes: immunoreactive, proliferative, differentiated and mesenchymal according to the classification criteria of OC molecular subtypes by the TCGA research team. Combined with HNRNPU expression data and OC typing data, we also found that high expression of HNRNPU was associated with the proliferative 
molecular subtype (Fig. 2D). Then, the GEO dataset GSE9891 was used to verify the results of the TCGA analysis, which also proved that high expression of HNRNPUis associated with the proliferative molecular subtype (Fig. 2E). Previous studies have shown that the proliferative molecular subtype is characterized by low expression of $\mathrm{OC}$ markers and overexpression of proliferation- and extracellular matrix (EMT)-related genes[6, 21, 31].

\section{HNRNPU silencing inhibits the proliferation of OC cells}

To study the effect of HNRNPU on the proliferation of OC cells, we used two HNRNPU-specific siRNAs to construct HNRNPU-knockdown cells. RT-PCR confirmed that siHNRNPU significantly downregulated HNRNPU mRNA expression in the OC cell line (Fig. 3A). In addition, Western blot analysis further verified that siHNRNPU downregulated the protein expression level of HNRNPU (Fig. 3B). CCK-8 analysis showed that HNRNPU silencing significantly reduced the number of OVCAR3 and ES2 cells (Fig. 3C). Compared with siNC transfection, HNRNPU silencing also significantly reduced the average number of colonies in the colony formation assay (Fig. 3D). In addition, an EdU assay was carried out to further verify the role of HNRNPU in the proliferation of OC cells. As shown in Fig. 3E, HNRNPU silencing significantly reduced the proportion of cells labeled with EdU. These results suggest that downregulation of HNRNPU may inhibit the proliferation of OC cells.

\section{HNRNPU silencing induces cell cycle arrest in OC}

To investigate whether the inhibition of OC cell proliferation by HNRNPU silencing was associated with cell cycle progression, cell cycle distribution was analyzed using flow cytometry. Compared with that in the siNC-transfected group, the proportion of OC cells transfected with siHNRNPU was significantly increased in the G1/S phase of the cell cycle (Fig. 4). In conclusion, the results indicated that HNRNPU might affect the proliferation of OC cells by blocking cell cycle progression.

\section{Discussion}

HNRNPS are a large family of RNA-binding proteins (RBPs) that have different molecular weights ranging from 34 to $120 \mathrm{kDa}$ and are named alphabetically from HNRNPA1 to HNRNPU14]. These proteins are involved in multiple aspects of nucleic acid metabolism, including alternative splicing, mRNA stabilization, and transcriptional and translational regulation[11]. The expression level of HNRNPs is altered in many types of cancer, indicative of their role in tumorigenesis[12]. Previous studies have shown that HNRNPs are closely related to the occurrence and development of many kinds of tumors[13, 19], including lung cancer[23], gastric cancer[10], and Merkel cell carcinoma[8]. In addition to their roles in cancer, many HNRNPs are also associated with neurodegenerative diseases, such as spinal muscular atrophy (SMA), amyotrophic lateral sclerosis (ALS), Alzheimer's disease (AD) and frontotemporal lobar dementia (FTLD) [3, 4, 25, 32].

HNRNPUis the largest molecule among the major members of the HNRNP RNA-binding protein family[18] and has the ability to bind RNA in vivo and double-stranded DNA in vitro to regulate gene expression at 
both the transcriptional and precursor RNA processing levels. In cancer-related research, HNRNPU has been demonstrated to interact with the tumor suppressor gene WT1 and proto-oncogene C-myc[29] and regulate the alternative splicing of the apoptosis regulatory protein Cas9[20], suggesting that it may be involved in tumorigenesis. In addition, disruption of the interaction of B23 with HNRNPU/HNRNPA1 leads to increased cellular sensitivity to apoptosis-inducing agents[34, 35]. Interestingly, the HNRNPU protein itself is cleaved during apoptosis[34], but there has not been direct evidence of the relationship between HNRNPU and tumorigenesis.

In this study, through the bioinformatic analysis of the genes in the AMP region in TCGA, we demonstrated for the first time that HNRNPU is closely related to the occurrence and development of OC. The results showed that AMP significantly upregulated the mRNA level of HNRNPU. Then, five independent datasets (GSE6008, GSE18520, GSE26712, GSE27651 and GSE29450) from the GEO database were meta-analyzed to confirm the high expression of HNRNPU in OC. Compared with that in nontumor samples, HNRNPU was mainly overexpressed ( $\left.S M D=1.27,95 \% \mathrm{Cl}=0.38-2.16, \mathrm{I}^{2}=81 \%\right)$ in OC samples. On the other hand, the overexpression of HNRNPU was also related to the subtype of OC categorized by the TCGA research team. The results showed that the overexpression of HNRNPU is mainly concentrated in proliferative OC. In addition, the GSE9891 dataset of the GEO database also confirmed that the group with high expression of HNRNPU was mainly enriched in the OC proliferative molecular subtype.

In addition, we established a cell model with HNRNPU downregulation to explore the role of HNRNPU in OC. After siRNA targeting HNRNPU was transfected into two cell lines, the expression of HNRNPU was downregulated, and proliferation was significantly inhibited in siHNRNPU-transfected OVCAR3 and ES2 cells compared with siNC-transfected cells, indicating that HNRNPU promotes the proliferation of OC cells. Flow cytometry analysis showed that downregulation of HNRNPU may inhibit the transition of OC cells from $\mathrm{G} 1$ phase to $\mathrm{S}$ phase. These results suggest that the effect of HNRNPUdownregulation on the proliferation of $\mathrm{OC}$ cells may be related to the regulation of the cell cycle.

\section{Conclusions}

In summary, the high expression of HNRNPU plays an important role in the occurrence and development of $\mathrm{OC}$ and is closely related to the prognosis of patients. Therefore, HNRNPU may be used as a therapeutic target for cancer in the future.

\section{Abbreviations}

OC: Ovarian cancer; FIGO I: early-stage; TCGA: The Cancer Genome Atlas; SCNAs: somatic copy number alterations; AMP: amplification; GEO: Gene Expression Omnibus; DEGs: differentially expressed genes; HNRNPU: heterogeneous nuclear ribonucleoprotein U; DMEM: Dulbecco's modified Eagle's medium; FBS: fetal bovine serum; siRNAs: small interfering RNAs; RT: reverse transcription; RT-PCR: Quantitative RT polymerase chain reaction; GAPDH: glyceraldehyde-3-phosphate dehydrogenase; PMSF: 
phenylmethylsulfonyl fluoride; EdU: 5-Ethynyl-2'-deoxyuridine; PBS: phosphate-buffered saline; RBPs:

RNA-binding proteins; SMA: spinal muscular atrophy; ALS: amyotrophic lateral sclerosis; AD: Alzheimer's disease; FTLD: frontotemporal lobar dementia; PI: propidium iodide.

\section{Declarations}

\section{Ethics approval and consent to participate:}

Not applicable.

\section{Consent for publication:}

Not applicable.

\section{Availability of data and materials:}

All data generated or analyzed during this study are included in this published article.

\section{Competing interests:}

The authors declare that they have no competing interests.

\section{Funding:}

This work was supported by the National Natural Science Foundation of China (Grant No. \#81760504 to Fen Fu); the Natural Science Foundation of Jiangxi Province (Grant No. \#20161ACB20022 to Fen Fu); the National Natural Science Foundation of China (Grant No. \#82060474 to Xiaoli Tang).

\section{Authors' contributions:}

FF conceived of the concept. LJ made substantial contributions to data collection and interpretation of results; LLL, TXL, FYP and FYY analyzed the data; LJ wrote the manuscript. LLL assisted with manuscript preparation. All authors read and approved the final manuscript.

\section{Acknowledgements:}

Thanks to Prof. Deng Libin for providing the technical help and writing assistance.

\section{References}

1. Alagpulinsa D, Ayyadevara S, Yaccoby S, Shmookler Reis RA, Cyclin-Dependent. Kinase Inhibitor, Dinaciclib, Impairs Homologous Recombination and Sensitizes Multiple Myeloma Cells to PARP Inhibition. Mol Cancer Ther. 2016;15(2):241-50.

2. Au-Yeung G, Lang F, Azar WJ, Mitchell C, Jarman KE, Lackovic K, et al. Selective Targeting of Cyclin E1-Amplified High-Grade Serous Ovarian Cancer by Cyclin-Dependent Kinase 2 and AKT Inhibition. 
Clin Cancer Res. 2017;23(7):1862-74.

3. Bebee TW, Dominguez CE, Samadzadeh-Tarighat S, Akehurst KL, Chandler DS. Hypoxia is a modifier of SMN2 splicing and disease severity in a severe SMA mouse model. Hum Mol Genet. 2012;21(19):4301-13.

4. Berson A, Barbash S, Shaltiel G, Goll Y, Hanin G, Greenberg DS, et al. Cholinergic-associated loss of hnRNP-A/B in Alzheimer's disease impairs cortical splicing and cognitive function in mice. EMBO Mol Med. 2012;4(8):730-42.

5. Bray F, Ferlay J, Soerjomataram I, Siegel RL, Torre LA, Jemal A. Global cancer statistics 2018: GLOBOCAN estimates of incidence and mortality worldwide for 36 cancers in 185 countries. CA Cancer J Clin. 2018;68(6):394-424.

6. Cancer Genome Atlas Research N. Integrated genomic analyses of ovarian carcinoma. Nature. 2011;474(7353):609-15.

7. Chen W, Zheng R, Baade PD, Zhang S, Zeng H, Bray F, et al. Cancer statistics in China, 2015. CA Cancer J Clin. 2016;66(2):115-32.

8. Chettouh H, Fartoux L, Aoudjehane L, Wendum D, Clapéron A, Chrétien Y, et al. Mitogenic insulin receptor-A is overexpressed in human hepatocellular carcinoma due to EGFR-mediated dysregulation of RNA splicing factors. Cancer Res. 2013;73(13):3974-86.

9. Ciriello G, Miller ML, Aksoy BA, Senbabaoglu Y, Schultz N, Sander C. Emerging landscape of oncogenic signatures across human cancers. Nat Genet. 2013;45(10):1127-33.

10. Dai P, Wang Q, Wang W, Jing R, Wang W, Wang F, et al. Unraveling Molecular Differences of Gastric Cancer by Label-Free Quantitative Proteomics Analysis. International journal of molecular sciences. 2016;17(1).

11. Dreyfuss G, Matunis MJ, Pinol-Roma S, Burd CG. hnRNP Proteins and the Biogenesis of mRNA. Annu Rev Biochem. 1993;62(1):289-321.

12. Geuens T, Bouhy D, Timmerman V. The hnRNP family: insights into their role in health and disease. Hum Genet. 2016;135(8):851-67.

13. Gonçalves V, Pereira J, Jordan P. Signaling Pathways Driving Aberrant Splicing in Cancer Cells. Genes. 2017:9(1).

14. Han SP, Tang YH, Smith R. Functional diversity of the hnRNPs: past, present and perspectives. Biochem J. 2010;430(3):379-92.

15. Jabbour-Leung N, Chen X, Bui T, Jiang Y, Yang D, Vijayaraghavan S, et al. Sequential Combination Therapy of CDK Inhibition and Doxorubicin Is Synthetically Lethal in p53-Mutant Triple-Negative Breast Cancer. Mol Cancer Ther. 2016;15(4):593-607.

16. Johnson S, Cruz C, Greifenberg A, Dust S, Stover D, Chi D, et al. CDK12 Inhibition Reverses De Novo and Acquired PARP Inhibitor Resistance in BRCA Wild-Type and Mutated Models of Triple-Negative Breast Cancer. Cell reports. 2016;17(9):2367-81. 
17. Kanska J, Zakhour M, Taylor-Harding B, Karlan BY, Wiedemeyer WR. Cyclin E as a potential therapeutic target in high grade serous ovarian cancer. Gynecol Oncol. 2016;143(1):152-8.

18. Kawano S, Miyaji M, Ichiyasu S, Tsutsui K, Tsutsui K. Regulation of DNA Topoisomerase llbeta through RNA-dependent association with heterogeneous nuclear ribonucleoprotein $\mathrm{U}$ (hnRNP U). J Biol Chem. 2010;285(34):26451-60.

19. Kędzierska H, Piekiełko-Witkowska A. Splicing factors of SR and hnRNP families as regulators of apoptosis in cancer. Cancer letters. 2017;396:53-65.

20. Kukalev A, Nord Y, Palmberg C, Bergman T, Percipalle P. Actin and hnRNP U cooperate for productive transcription by RNA polymerase II. Nat Struct Mol Biol. 2005;12(3):238-44.

21. Leong HS, Galletta L, Etemadmoghadam D, George J, Australian Ovarian Cancer S, Kobel M, et al. Efficient molecular subtype classification of high-grade serous ovarian cancer. J Pathol. 2015;236(3):272-7.

22. Li W, Liu Z, Liang B, Chen S, Zhang X, Tong X, et al. Identification of core genes in ovarian cancer by an integrative meta-analysis. J Ovarian Res. 2018;11(1):94.

23. Liu X, Zhou Y, Lou Y, Zhong H. Knockdown of HNRNPA1 inhibits lung adenocarcinoma cell proliferation through cell cycle arrest at G0/G1 phase. Gene. 2016;576:791-7.

24. Marcus CS, Maxwell GL, Darcy KM, Hamilton CA, McGuire WP. Current approaches and challenges in managing and monitoring treatment response in ovarian cancer. J Cancer. 2014;5(1):25-30.

25. Neumann M, Sampathu DM, Kwong LK, Truax AC, Micsenyi MC, Chou TT, et al. Ubiquitinated TDP-43 in frontotemporal lobar degeneration and amyotrophic lateral sclerosis. Science. 2006;314(5796):130-3.

26. Patch AM, Christie EL, Etemadmoghadam D, Garsed DW, George J, Fereday S, et al. Whole-genome characterization of chemoresistant ovarian cancer. Nature. 2015;521(7553):489-94.

27. Siegel RL, Miller KD, Jemal A. Cancer statistics, 2020. CA Cancer J Clin. 2020;70(1):7-30.

28. Smith RA, Manassaram-Baptiste D, Brooks D, Doroshenk M, Fedewa S, Saslow D, et al. Cancer screening in the United States, 2015: a review of current American cancer society guidelines and current issues in cancer screening. Cancer J Clin. 2015;65(1):30-54.

29. Spraggon L, Dudnakova T, Slight J, Lustig-Yariv O, Cotterell J, Hastie N, et al. hnRNP-U directly interacts with WT1 and modulates WT1 transcriptional activation. Oncogene. 2007;26(10):1484-91.

30. Torre LA, Trabert B, DeSantis CE, Miller KD, Samimi G, Runowicz CD, et al. Ovarian cancer statistics, 2018. CA Cancer J Clin. 2018;68(4):284-96.

31. Tothill RW, Tinker AV, George J, Brown R, Fox SB, Lade S, et al. Novel molecular subtypes of serous and endometrioid ovarian cancer linked to clinical outcome. Clin Cancer Res. 2008;14(16):5198-208.

32. Vance C, Rogelj B, Hortobagyi T, De Vos KJ, Nishimura AL, Sreedharan J, et al. Mutations in FUS, an RNA processing protein, cause familial amyotrophic lateral sclerosis type 6. Science. 2009;323(5918):1208-11. 
33. Vijg J. Somatic mutations, genome mosaicism, cancer and aging. Curr Opin Genet Dev. 2014;26:141-9.

34. Vizlin-Hodzic D, Runnberg R, Ryme J, Simonsson S, Simonsson T. SAF-A forms a complex with BRG1 and both components are required for RNA polymerase II mediated transcription. PloS one. 2011;6(12):e28049.

35. Yao Z, Duan S, Hou D, Wang W, Wang G, Liu Y, et al. B23 acts as a nucleolar stress sensor and promotes cell survival through its dynamic interaction with hnRNPU and hnRNPA1. Oncogene. 2010;29(12):1821-34.

\section{Figures}

A

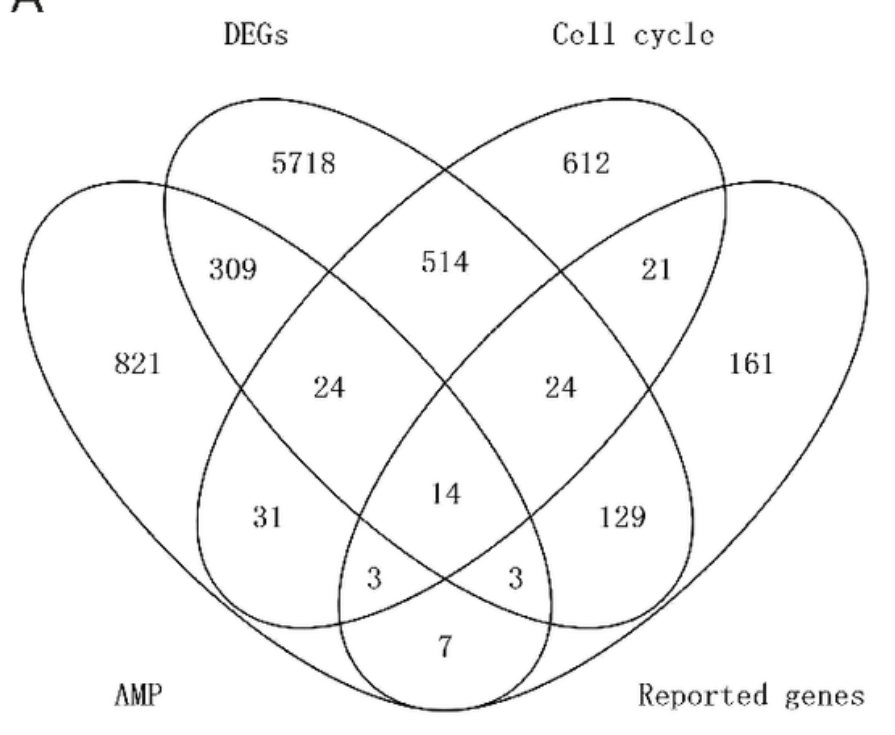

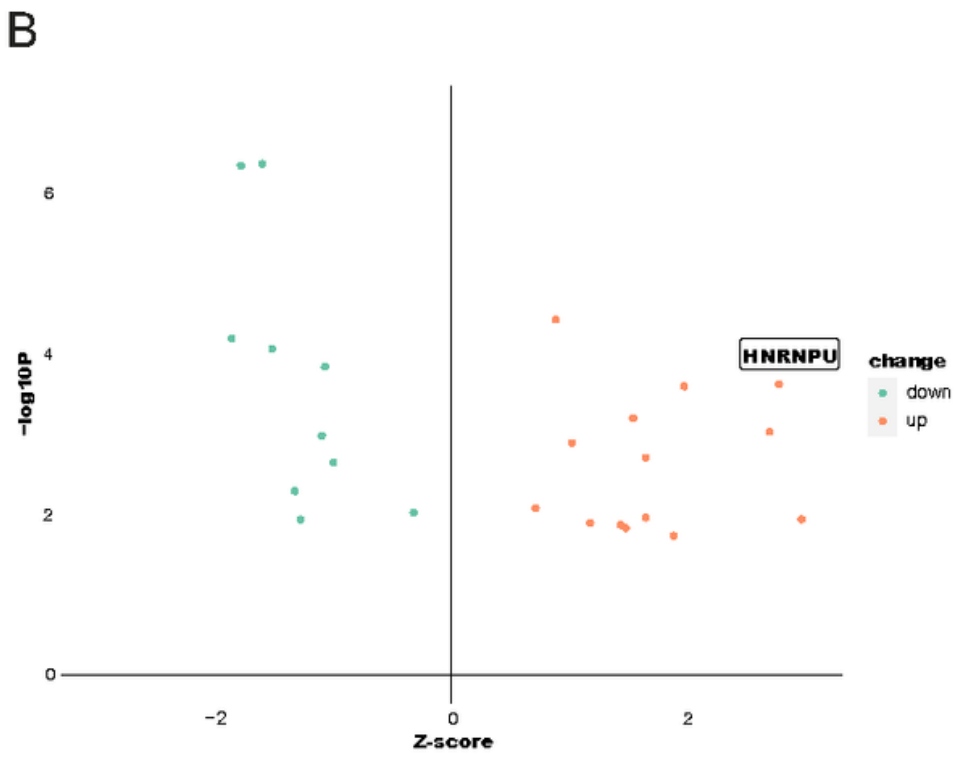

\section{Figure 1}

The process of target gene HNRNPU selection. A. The diagram shows cell cycle-related genes involved in AMP. We detected 24 unreported genes. B. The scatter plot shows that among the 24 genes, HNRNPU was the most strongly affected (P-value $\mathbb{0} .0002$, Z-score $\ 2.77$ ). 
A

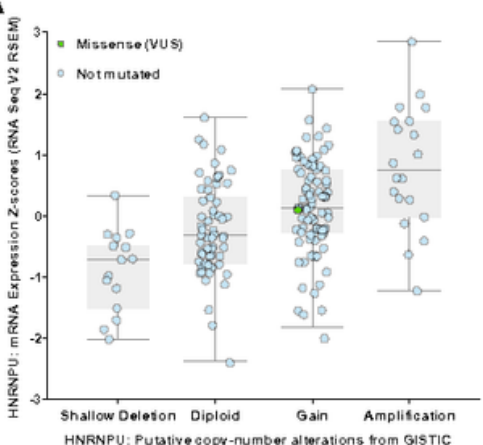

C

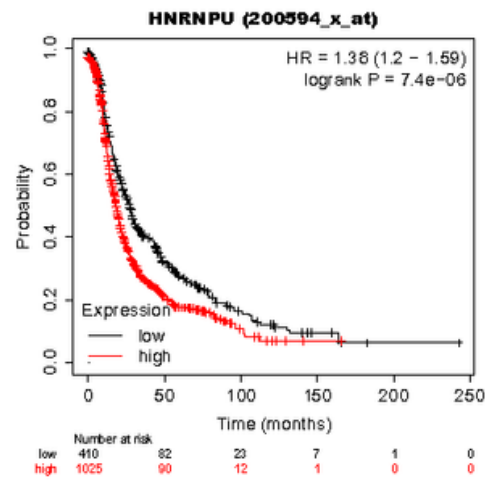

B

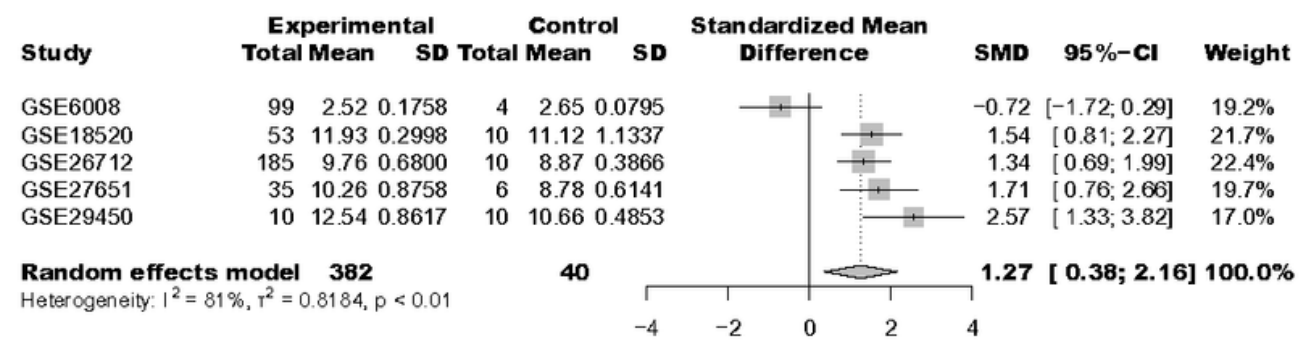

D

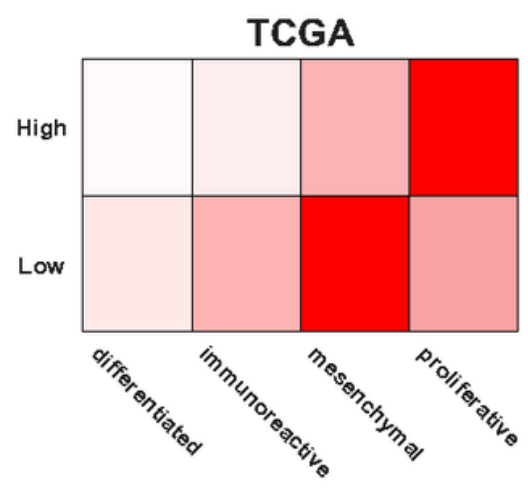

$E$

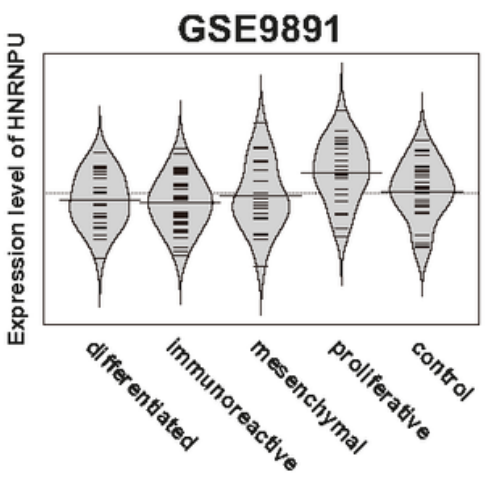

Figure 2

HNRNPU expression correlates with OC SCNAs, prognosis and OC subtypes. A. The relationship between HNRNPU expression and SCNAs. Copy number amplification upregulates HNRNPU gene expression. B. Meta-analysis comparing HNRNPU expression in OC and para-carcinoma tissues based on five GEO datasets. The forest plot shows that HNRNPU expression is upregulated in OC. C. The association of HNRNPU expression with patient survival based on 614 OC patients. The Kaplan-Meier plot illustrates that a high expression of HNRNPU is related to a worse prognosis of OC. D. The relationship between HNRNPU expression and OC subtypes based on TCGA data. E. The relationship between HNRNPU expression and OC subtypes based on the GSE9891 dataset. 


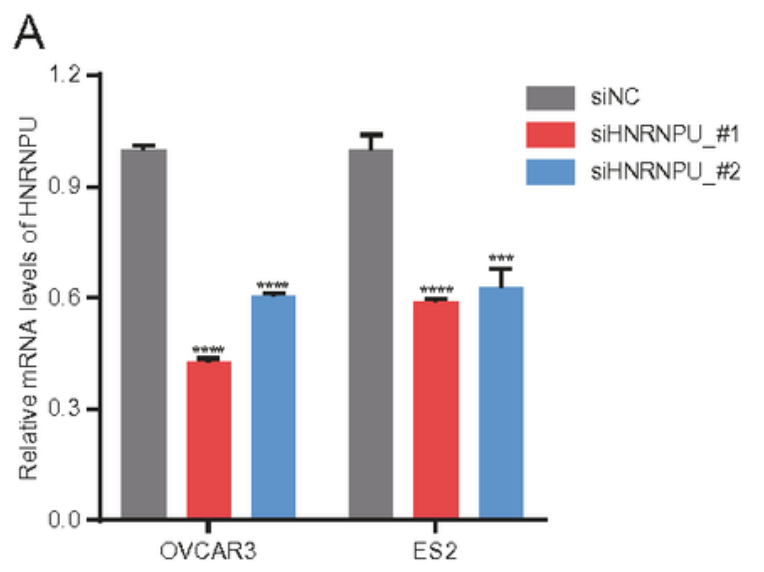

B
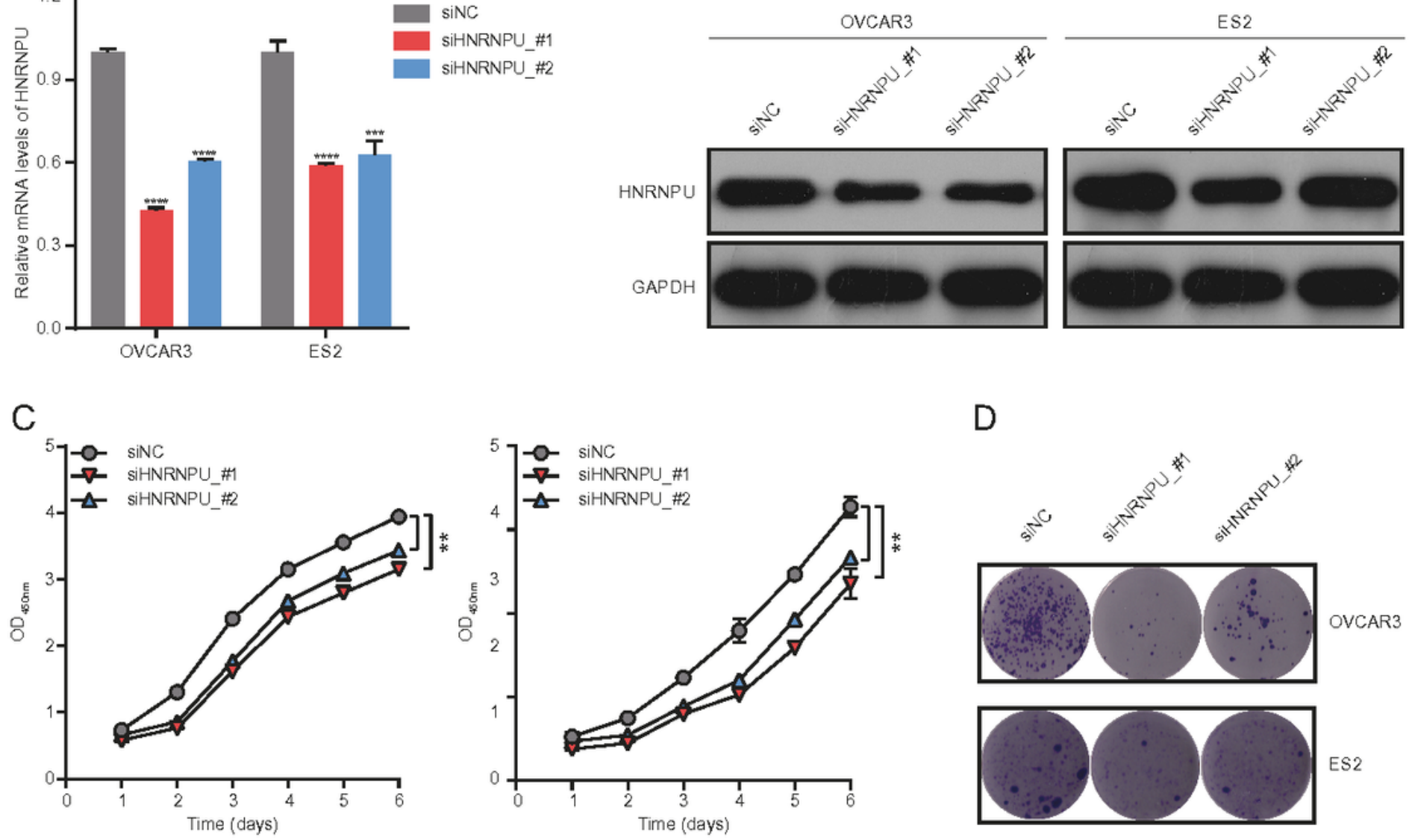

D

$\mathrm{E}$
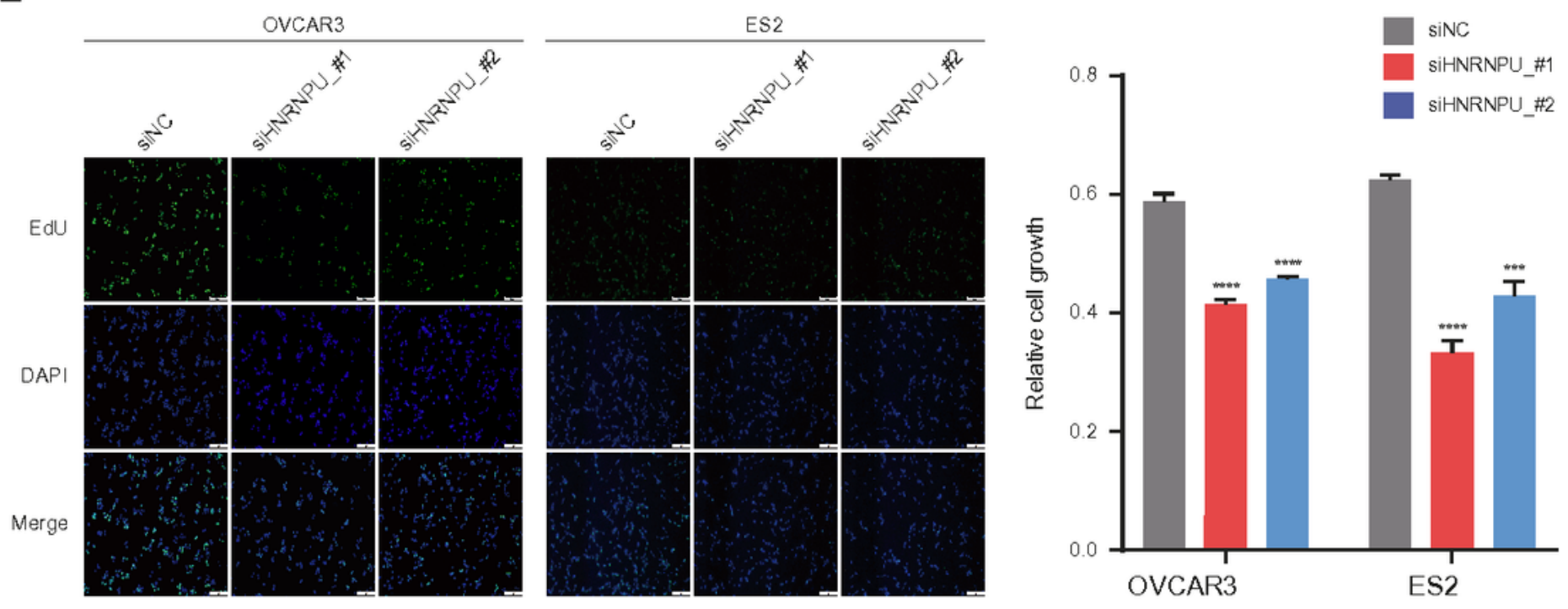

\section{Figure 3}

Effects of HNRNPU silencing on the proliferation of OC cells. A. RT-PCR of the indicated OC cells transfected with siNC, siHNRNPU_\#1 or siHNRNPU_\#2. The capability of different siRNAs to downregulate HNRNPU mRNA expression. B. Western blot of the indicated OC cells transfected with siNC, siHNRNPU_\#1 or siHNRNPU_\#2. The capability of different siRNAs to downregulate HNRNPU protein expression. C. CCK-8 assays revealed that HNRNPU knockdown significantly reduced the growth rate. D. 
HNRNPU knockdown reduced the colony number in the colony formation assay. $E$. The proliferation rate of OVCAR3 and ES2 cell lines at $48 \mathrm{~h}$ after HNRNPU knockdown. After transfection of HNRNPU siRNA into OVCAR3 and ES2 cells, HNRNPU expression was downregulated and proliferation was dramatically inhibited compared to those in siNC-transfected cells. ${ }^{\star \star} p<0.01,{ }^{* \star *} p<0.001,{ }^{* \star \star *} p<0.0001$ versus siNC group.
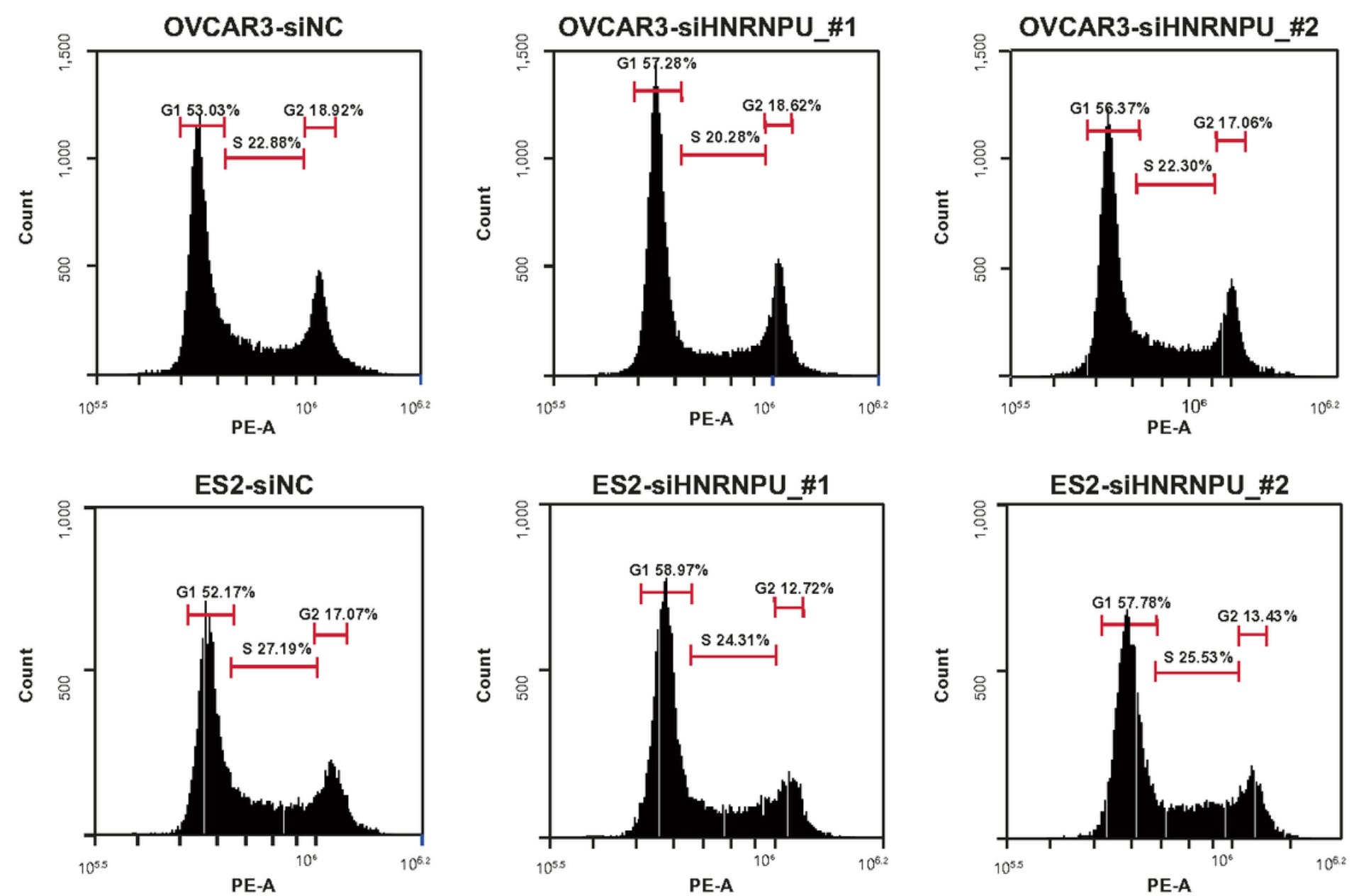

Figure 4

Effects of HNRNPU silencing on the cell cycle. OVCAR3 and ES2 cells were treated with siNC, siHNRNPU_\#1 or siHNRNPU_\#2 for $24 \mathrm{~h}$, and the isolated nuclei stained with propidium iodide (PI) were analyzed by flow cytometry. 